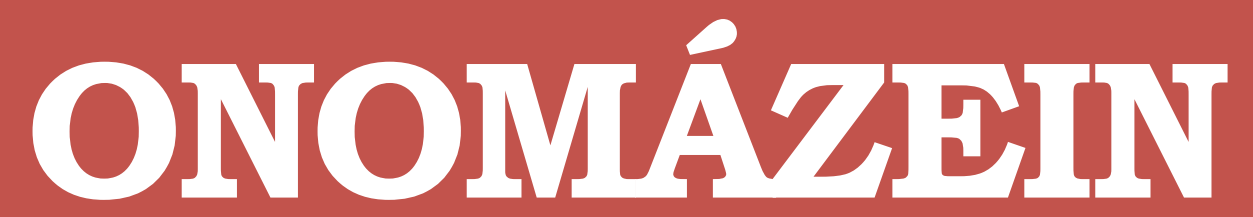

Revista de lingüística, filología y traducción

\title{
El lenguaje inclusivo en el discurso político español: un análisis del lenguaje de los políticos españoles antes y después de una campaña electoral
}

Non-sexist language in Spanish political discourse: an analysis of the language of Spanish politicians before and after an electoral campaign

\footnotetext{
María del Carmen Lagares Carretero

Universidad de Estocolmo

Suecia
} 


\section{Resumen}

El presente estudio analiza el lenguaje de Mariano Rajoy del Partido Popular, Pedro Sánchez del PSOE, Albert Rivera de Ciudadanos, Pablo Iglesias de Podemos y Alberto Garzón de Izquierda Unida, antes y después de las elecciones del veinte de diciembre de 2015, según seis guías feministas sobre el uso correcto del lenguaje inclusivo. Las categorías lingüísticas que se han analizado son los recursos léxicos, los recursos gramaticales y las formas de tratamiento. El objetivo de este estudio es comprobar si los representantes políticos adecúan su discurso según el lenguaje inclusivo y si estos varían su lenguaje dependiendo de si sucede en un contexto formal o un contexto informal o si tuvieron lugar antes o después de las elecciones. El corpus está formado por discursos preelectorales que se obtienen de dos programas televisivos y por discursos postelectorales que se obtienen de sesiones plenarias en el Congreso de los Diputados. Una vez analizados, todos los discursos se comparan entre ellos para comprobar en qué momento se usa un lenguaje inclusivo y qué político hace uso de este. En los resultados se puede observar que Mariano Rajoy y Pedro Sánchez hacen uso o no del lenguaje inclusivo dependiendo del contexto y del momento de las elecciones mientras que Albert Rivera y Pablo Iglesias no usan el lenguaje inclusivo en ningún contexto ni tampoco antes y después de las elecciones. En cambio, Alberto Garzón usa el leguaje inclusivo tanto en el contexto formal como el informal y antes y después de las elecciones. Por tanto, se concluye que Alberto Garzón es el candidato que usa el lenguaje inclusivo.

Palabras clave: lenguaje inclusivo; recursos inclusivos; recursos léxicos; recursos gramaticales; lenguaje sexista.

\section{Abstract}

The current study analyses the speech of the five most important political parties in Spain, Mariano Rajoy who belongs to Partido Popular, Pedro Sánchez to PSOE, Albert Rivera to Ciudadanos, Pablo Iglesias to Podemos and Alberto Garzón to Izquierda Unida, before and after the elections which took place on December the 20th in 2015 according to six handbooks about how to use proper non-sexist language. The aim of this study is to test whether politicians adapt their speech according to non-sexist language and whether these speeches vary according to formal and informal situations and the moment in which the speeches took 
place, that is, before or after the elections. The pre-electoral speeches are obtained from two tv programmes and the post-electoral speeches are obtained from the Parliament. Once all the speeches have been analysed, they are compared to confirm when non-sexist language is used, and which politicians use non-sexist language. Regarding the results, Mariano Rajoy and Pedro Sánchez use non-sexist language depending on the context and the situation within the elections whereas Albert Rivera and Pablo Iglesias do not use non-sexist language. However, Alberto Garzón uses non-sexist language in formal and informal context and before and after the elections. Therefore, we come to the conclusion that Alberto Garzón is the politician who uses non-sexist language.

Keywords: non-sexist language; inclusive means; lexical means; grammatical means; sexist language. 


\section{Introducción}

Según Eulàlia Lledó, "lo que no se nombra no existe o se le está dando carácter de excepción” (Lledó, 2004: 22). Con esta afirmación parte la teoría del lenguaje inclusivo y su idea de que el lenguaje es una herramienta que mantiene el androcentrismo y el sexismo en la sociedad, por lo que se considera que una de las posibles vías con las que se puede llegar a alcanzar una sociedad libre de machismo es a través del lenguaje y sus hablantes.

Sin embargo, son muchos los lingüistas y expertos que se han posicionado en contra de este cambio en la lengua. La Real Academia de la Lengua Española nos dice que el masculino es un género no marcado que se debe usar para incluir a ambos sexos con independencia del número de individuos que se encuentren presentes (RAE, "Preguntas frecuentes": "Los ciudadanos y las ciudadanas, los niños y las niñas"). Asimismo, Ignacio Bosque, miembro de la RAE, desacreditó a las guías sobre el uso del lenguaje inclusivo afirmando que estas guías no están escritas por lingüistas especializados, sino que en ellas aparecen las decisiones que los hispanohablantes toman sobre la lengua sin conocimiento de esta (Bosque, 2012: 1-2). A pesar de estas críticas, la idea de la necesidad de usar un lenguaje inclusivo cada vez está asentándose más en nuestra sociedad y cuenta con apoyos de reconocidas lingüistas como Eulàlia Lledó, Mercedes Bengoechea e Inés Fernández-Ordóñez, también miembro de la RAE, que se muestra partidaria de aceptar los cambios en la lengua y respetar los cambios que proponen los hablantes (Ruiz, 2016). Además de estas lingüistas, grupos feministas en España y partidos políticos, como Podemos y su Proposición no de ley de 2016 para usar el lenguaje inclusivo en el Congreso de Los Diputados, están promoviendo la idea del lenguaje inclusivo como recurso para promover la igualdad y están llevando el debate sobre el lenguaje a los plenos del Congreso.

Por estos motivos, sostenemos que no hay mejor manera de analizar a una sociedad que a través de sus representantes políticos. El objetivo de este trabajo es analizar el uso del lenguaje de cinco candidatos a la presidencia del gobierno español que representan a las cinco fuerzas políticas más importantes en España antes y después de las elecciones generales en diciembre de 2015. El lenguaje que se ha analizado corresponde a los discursos concedidos en entrevistas televisivas antes de las elecciones y los discursos en los debates en el Congreso de los Diputados una vez finalizadas las elecciones. El análisis del lenguaje se enfoca, principalmente, en la visibilidad concedida a las mujeres, la frecuencia con la que se usan los recursos inclusivos y la temática del contexto en la que se nombra a las mujeres. También se presta atención a la ideología y la edad de los cinco candidatos como factores que determinan si se usa o no el lenguaje inclusivo.

Para nuestro estudio hemos usado seis guías sobre el uso del lenguaje inclusivo publicadas en España entre 1990 y 2012 que se pueden aplicar al lenguaje oral y escrito, a la vez que se pueden usar en registros coloquiales y cultos. Concretamente, nos referimos a las 
siguientes guías: La representación del femenino y el masculino en el lenguaje, publicada por NOMBRA en 1995; Recomendaciones para un uso no sexista del lenguaje, publicada por la UNESCO en 1990 y escrita originalmente en inglés y francés; Guía para la utilización no sexista del lenguaje, publicada en 2006 por la Asociación Cultural Simone de Beauvoir; Guía para la revisión del lenguaje desde la perspectiva de género, escrita por Mercedes Bengoechea en 2002; Lenguaje inclusivo, escrito por Eulàlia Lledó en 2012; Guía para el uso no sexista del lenguaje, publicada por la Universitat Autònoma de Barcelona en 2011; y, finalmente, Manual de comunicación no sexista, publicado por el Ajuntament de Terrassa el 21 de junio de 2004. En estas guías aparecen una serie de recursos inclusivos para evitar el sexismo y el androcentrismo en el lenguaje y así crear un lenguaje igualitario en el que la mujer no esté relegada a un segundo plano o ausente. Algunos de los recursos más significantes que se promueven es evitar el uso del masculino genérico para incluir a las mujeres, el cual se puede intercambiar por el uso de sustantivos neutros o por el uso de las formas dobles de los artículos y los sustantivos. Como referencia para el lenguaje parlamentario y político, nos hemos basado en los artículos de Damián Moreno, "Los alcaldes y alcaldesas: referencia a personas y género en el lenguaje parlamentario andaluz" (2012); Esperanza R. Alcaidea, “El ‘yo’ de los políticos ¿cuestión de género?” (2009); Armando Francesconi, “El sexismo en el lenguaje político español e italiano” (2012); y, finalmente, en el artículo de Catalina Rodríguez Fuentes, “Argumentación, (des)cortesía y género en el discurso parlamentario" (2013).

\section{Estado de la cuestión}

Se podría considerar el lenguaje inclusivo como un tema reciente en la sociedad española, aunque, en realidad, el movimiento por el uso de un lenguaje no sexista comenzó en la década de 1960 como una rama de la lingüística dentro de las ciencias sociales. Este pensamiento surgió como una propuesta del feminismo para acabar con la invisibilidad de la mujer en el habla y la escritura y contra el androcentrismo. En lo que respecta a España, no se sabe con exactitud cuándo se empezó a hablar de lenguaje inclusivo. Se podría tomar como fecha de inicio la década de 1990, cuando la Comisión Asesora sobre Lenguaje del Instituto de la Mujer, también conocida como NOMBRA, redactó un manual en 1995 llamado La representación del femenino y el masculino en el lenguaje. El manual explica qué es el lenguaje sexista y a su vez ejemplifica cómo se debe utilizar el lenguaje para evitar el sexismo lingüístico. A pesar de que la idea de lenguaje inclusivo lleva varias décadas en España, no ha sido hasta mediados del 2007 cuando la prensa empezó a hacerse eco del cambio que se quería implementar en la lengua española, y para ser más precisos, no hace más de ocho años que este tema se ha convertido en uno de los temas más hablados tanto en prensa como en el día a día de la ciudadanía española. Es importante decir que, a pesar de ser un movimiento que se creó con la idea de acabar con las desigualdades de género en la sociedad y no solo en la lengua, han surgido numerosos detractores del lenguaje inclusivo por parte tanto de hombres como de 
mujeres, y especialmente por parte de los académicos de la Real Academia de la Lengua Española. Otros problemas que se han desarrollado respecto al lenguaje inclusivo es el propio concepto de lenguaje inclusivo en sí, es decir, la mayoría de las personas tiene una idea errónea sobre cómo se debe utilizar el lenguaje no sexista o cómo se debería escribir para incluir tanto al género masculino como al género femenino. Es muy común leer y oír en la prensa ideas contrarias al lenguaje inclusivo o ideas que se le atribuyen directamente al mismo sin ser del todo correctas. Esta actividad está causando confusión entre la población y está haciendo que expertos lingüistas tomen una posición contraria a la misma, como es el caso de Ignacio Bosque ("Sexismo lingüístico y visibilidad de la mujer”, 2012) y Arturo Pérez-Reverte (“No siempre limpia y da esplendor”, 2016).

Por esta razón, este trabajo nace con la idea de comparar los discursos políticos de los principales partidos en España debido a la gran importancia que los propios políticos le han dado al feminismo durante las campañas electorales. El interés que se está otorgando al feminismo en los últimos años Ilama bastante la atención en lo que respecta a política ya que el movimiento feminista ha existido en España desde el siglo XIX, con personajes tan importantes como Emilia Pardo Bazán y Concepción Arenal, y el siglo XX, en el que destaca la figura de Clara Campoamor (UNED, 2012). Este interés se puede tomar como una forma de propaganda cuyo fin podría ser más el de captar votantes que el de propiciar un verdadero cambio social. En cuanto a material relacionado con el lenguaje inclusivo en el discurso político en España, se han encontrado trabajos de fin de carrera y artículos en los que se analiza un mismo partido político o se establecen pautas sobre cómo debe ser un lenguaje político no sexista. Como, por ejemplo, "Los alcaldes y alcaldesas: referencia a personas y género en el lenguaje parlamentario andaluz", escrito por Damián Moreno Benítez en 2012, y Sexismo lingüístico y lenguaje político: análisis del lenguaje inclusivo en el discurso de Podemos, escrito por Olalla Martínez Iglesias en 2015. Sin embargo, no se han encontrado tesis que analicen o comparen el lenguaje de los representantes de los principales partidos políticos en España. Por este motivo, consideramos que hay un vacío de conocimiento respecto al objeto de este trabajo, y debido a este mismo motivo hemos analizado el discurso de los principales políticos españoles para ver si hacen un uso no sexista del lenguaje que contribuya a un cambio en la sociedad.

\section{Los representantes políticos}

Los cinco candidatos escogidos son todos hombres españoles con edades comprendidas entre los 30 y los 60 años. Estos candidatos son Mariano Rajoy, de 60 años y secretario general del Partido Popular; Pedro Sánchez, de 46 años y secretario general del Partido Socialista Obrero Español; Albert Rivera, de 36 años y secretario general de Ciudadanos; Pablo Iglesias, de 37 años y secretario general de Podemos, y, finalmente, Alberto Garzón, con 30 años y secretario general de Izquierda Unida. 


\section{Preguntas de investigación e hipótesis}

Este estudio parte con el objetivo de analizar el lenguaje de los cinco representantes políticos más votados en España. En concreto, nuestro trabajo quiere responder las siguientes preguntas:

1) ¿Usan los representantes políticos el mismo tipo de lenguaje antes y después de las elecciones?

2) ¿influye el tipo de contexto en el lenguaje de los representantes políticos?

3) ¿Varían los representantes políticos su lenguaje dependiendo del tema sobre el que se hable?

La primera hipótesis con la que se parte es que los políticos no utilizan siempre el lenguaje inclusivo, y en el caso de que lo hagan, su uso será más frecuente antes de las elecciones. La segunda hipótesis propone que encontraremos un lenguaje no sexista puesto que los políticos moderarán su discurso para adaptarlo a las ideas feministas y, a su vez, captar votos. No obstante, una vez pasadas las elecciones, puede que el lenguaje usado varíe respecto al que utilizaban durante la campaña electoral. Con nuestra última hipótesis pensamos que en situaciones formales y en las que los políticos hablen siguiendo un guion previamente escrito y ensayado para la ocasión, el lenguaje que se utilice será inclusivo, pero en situaciones informales en las que no hayan preparado lo que van a decir ni puedan seguir ningún guion, el lenguaje cambiará a uno más tradicional o menos inclusivo.

\section{Método y materiales}

Como materiales hemos utilizado las guías sobre el correcto uso del lenguaje inclusivo que han servido para analizar los discursos antes y después de las elecciones. Primero se ha observado cuáles son las principales características que debe tener un discurso para que se considere inclusivo. Posteriormente, se eligieron los discursos políticos y se transcribieron. Una vez que todos los discursos fueron transcritos, se dividieron para facilitar el análisis en dos apartados, los que se dieron antes de las elecciones y después de las elecciones, y dentro del apartado de antes de las elecciones, los discursos se separaron en aquellos que tuvieron lugar en un contexto formal y aquellos que ocurrieron en un contexto informal. Los discursos de después de las elecciones solo ocurren en un contexto formal. En cuanto al análisis del lenguaje de los políticos, hemos optado por analizar su lenguaje según qué recursos usen acorde al tema del que se esté hablando para observar a qué nivel y con qué frecuencia los políticos usan los recursos no sexistas de la lengua y logran, o no, un discurso con un lenguaje inclusivo.

\section{Corpus}

El corpus está formado por las transcripciones de los discursos dados por los representantes políticos, los cuales son tres discursos por cada uno de ellos. En total, se han elegido quince discursos que han tenido lugar en tres situaciones diferentes, pero solo hemos analizado 
fragmentos con una duración de entre diez y quince minutos y que son relevantes para el resultado del análisis. Del mismo modo, los discursos se dividen en dos grupos: contextos formales y contextos informales. Para este estudio, entendemos el contexto formal como aquella situación en la que el lenguaje utilizado se caracteriza por la seriedad del momento y el respeto por la jerarquía de los hablantes, los cuales hacen uso de un vocabulario amplio, especializado y muy preciso, usan frases largas y completas y la información que se expone aparece de forma estructurada y lógica. En cambio, entendemos por contexto informal aquella situación en la que los hablantes no consideran importante la jerarquía, se da en un ambiente de más confianza y no se cuida tanto el lenguaje, es decir, los hablantes usan repeticiones y formas redundantes, no se suele utilizar las formas de "usted", el vocabulario es menos específico con abundantes repeticiones y las frases son más cortas y no tan estructuradas (Enciclopedia Online, 2018). En los fragmentos del contexto formal analizados, los principales representantes de los cinco partidos políticos tratan temas de diferente índole, como, por ejemplo, economía, proyectos de leyes, inmigración e igualdad social durante una sesión plenaria en el Congreso de los Diputados. Lo que se pretende al analizar temas relacionados no solo con el feminismo es comparar si las personas que hablan utilizan el mismo lenguaje en relación con el asunto que están tratando o varían según el objeto de debate. En el contexto informal, los discursos que se han transcrito tienen lugar en el programa televisivo La Sexta Noche, retransmitido por la cadena española La Sexta. Se ha considerado este programa como contexto informal debido a que los políticos argumentan con las personas presentes en el programa de manera cordial y desenfadada. Se tutean o usan sus nombres y el lenguaje que se utiliza no usa un vocabulario específico, sino más bien coloquial y fácil de comprender para toda la ciudadanía. En el contexto formal, los discursos elegidos provienen del programa televisivo Los Desayunos de TVE, que se retransmite en la cadena pública española Televisión Española, y de las sesiones plenarias del Congreso de los Diputados de Madrid. Se han catalogado estos contextos como formales debido a la jerarquía que existe entre los participantes en el programa televisivo y los miembros del Congreso. Esta misma jerarquía se hace visible en la forma en la que los hablantes se dirigen entre ellos; por ejemplo, se tratan de "señor" o "señora" y no usan sus nombres de pila a no ser que vaya acompañado de una expresión formal. Además, el lenguaje que se utiliza está bien formulado y estructurado, con un vocabulario concreto y específico que se aleja de la norma coloquial.

\section{Análisis y resultados}

\section{Debates preelectorales en el contexto informal}

En el contexto informal observamos que el lenguaje más cercano a considerarse lenguaje inclusivo es el del político Alberto Garzón dado que es el que más visibilidad otorga a las mujeres y mantiene un tono neutro en la mayor parte de su discurso. Además de citar directamente a las mujeres junto a los hombres y no relegarlas a un segundo plano. Seguidamente, 
se encuentra Mariano Rajoy, que a pesar de no citar a las mujeres con la misma relevancia que a los hombres y hacer uso del masculino genérico en repetidas ocasiones, es el que también mantiene un tono más neutro durante su discurso gracias a los sustantivos genéricos, por lo que se incluye a hombres y mujeres en esa parte de su discurso. El lenguaje de Mariano Rajoy se encuentra en una posición en la que no se incluye dentro del lenguaje inclusivo, pero tampoco muestra las mismas características que el de los candidatos restantes que sí presentan un lenguaje excluyente. Por esta razón, hemos considerado que Pedro Sánchez, Pablo Iglesias y Albert Rivera no usan lenguaje inclusivo al no presentar recursos inclusivos suficientes que incluyan a la mujer y la visibilice. De igual modo, observamos que las menciones a la mujer por parte de estos representantes políticos son escasas. Además de estos motivos, la principal razón por la que se ha decidido situar a estos candidatos fuera del lenguaje inclusivo la encontramos en su forma de expresarse sobre las mujeres al mencionar la violencia machista. Únicamente Garzón nombra a las mujeres de forma directa, y en menor medida Rajoy, mientras que el resto de los candidatos dejan de usar el masculino genérico para usar sustantivos genéricos para hablar de un tema que concierne a las mujeres principalmente, por lo que se está excluyendo a la mujer en un momento en el que la protagonista del lenguaje debería ser ella. Por esta razón, consideremos que Garzón es el representante político que usa un lenguaje inclusivo en el contexto informal. En las siguientes oraciones se pueden apreciar algunos fragmentos de los discursos obtenidos en el contexto informal en los que se observa el uso de los recursos léxicos de los representantes políticos:

Alberto Garzón:

1) Una parte del esfuerzo que yo estoy haciendo, como muchos otros compañeros y compañeras, es tratar de unir a la izquierda fundamentalmente porque pensamos (se omite el sujeto) ... [... ... ni las personas, sino la gente, las clases populares a las que pertenecemos.

2) Lo que tenemos que hacer también es un elemento cultural, que es introducir el feminismo a nuestras vidas. Una cultura que asumamos todos y todas que somos hombres y mujeres igual y que no se puede permitir la opresión de la una por la otra.

Aunque AG usa el recurso de las formas dobles para visibilizar a las mujeres del partido, tendría que haber concordado en femenino los determinantes y así no incluir a compañeras dentro de los determinantes masculinos. En el segundo ejemplo AG no solo está enfatizando el papel del hombre y de la mujer en este asunto, sino que también está visibilizando a la mujer, y, además, de entre los políticos estudiados, es el único que habla de feminismo en sus respuestas.

Mariano Rajoy:

3) Aún hay gente que es capaz de liquidar la vida de otra persona, de machacarla, de atentar contra sus derechos y contra lo que es la esencia de una persona, sobre todo les pasa a las mujeres. 
4) Pero nosotros hemos apartado a todas esas personas que ... [... ... y muchas de estas personas han sido juzgados, algunos condenados, otros han sido absueltos.

En este fragmento podemos observar cómo no se nombra directamente a las mujeres, aunque se diga que este problema les ocurre a ellas o que todas las personas que trabajan en ese lugar fuesen mujeres. Es decir, está utilizando un recurso para incluir a hombres y mujeres a la vez que se afirma que solo se está hablando de mujeres, por lo que la mujer aparece como un apéndice del hombre en lugar de un ente independiente (Bengoechea, 2012: 10). Resulta interesante que cuando se está hablando sobre mujeres y los problemas que ellas sufren, MR hable sobre ellas de una forma neutra, mientras que para hablar de otros temas utilice en su mayoría el masculino como genérico como se aprecia en el segundo ejemplo. La concordancia de los adjetivos no coincide en género con el sustantivo al que acompañan y, en lugar de usar el femenino en concordancia con personas, opta por usar el masculino.

Pedro Sánchez:

5) El que quiera votar en el extranjero lo que tiene que hacer es pedirlo, rogar el voto.

6) Mi compromiso es recuperar ese derecho a la cotización en la seguridad social de los cuidadores no profesionales que sobre todo son mujeres.

Al igual que en el caso de MR, PS decide usar el masculino genérico, como en el primer ejemplo, donde se podría haber sustituido El que quiera votar por la persona que quiera votar, otorgándole así un sentido más neutro e inclusivo. Además, nos Ilama la atención también que PS prefiera usar el nombre de una profesión en masculino y añadirle después la palabra mujer para especificar de quién se habla en lugar de usar las formas dobles de los artículos delante de cuidadores y así evitar especificar de qué personas se está hablando.

Albert Rivera:

7) La patria para mí son los ciudadanos españoles, y además usted que es autónomo, yo soy hijo de autónomos, nieto de autónomos, sobrino de autónomos, y de autónomos algunos en paro.

8) No podemos trasladar a los médicos ni a las mujeres esas situaciones.

En esta respuesta, AR podría haber evitado el androcentrismo en el lenguaje (NOMBRA, 1995: 25) utilizando el pueblo españo/ y así también incluir a todas las personas que trabajen y vivan en España. Es cierto que la palabra autónomo no tiene una forma neutra que se pueda usar como sinónimo y sustituir la forma masculina por las formas dobles sonaría muy repetitivo. Aún así, se podría incluso simplificar esa misma frase usando la palabra persona para expresar la misma idea de una forma inclusiva. Por tanto, quedaría como soy hijo, nieto y sobrino de personas autónomas. En el segundo ejemplo hubiese sido más inclusivo utilizar el personal 
médico y las pacientes debido a que con esta interpretación parece estar descartando que las mujeres puedan ser profesionales como los hombres y está creando una diferencia de rangos sociales. Además, sobre este asunto, AR omite el uso del masculino genérico y comienza a usar personas y gente a pesar de que el aborto sea un asunto que solo concierne a las mujeres.

Pablo Iglesias:

9) Para nosotros es un honor haber aplicado la paridad de manera estricta en nuestras elecciones primarias.

10) Pues nosotros estamos con los autónomos, estamos con los pequeños y medianos empresarios y estamos con la gente que tiene que trabajar para vivir.

En el primer ejemplo se podría haber usado para el partido en lugar de nosotros y obtener una frase más inclusiva. Sin embargo, puede que el uso del masculino genérico en esta ocasión sea una herramienta para enfatizar a los miembros de su partido y así diferenciarse del resto de partidos políticos. En el segundo ejemplo nos encontramos una característica que no aparece en los discursos de MR, PS y AR. En ellos se menciona a estas personas como pequeñas y medianas empresas, la cual es la forma correcta de nombrar a las empresas. Sin embargo, el modo en el que PI se refiere a ellas resulta singular ya que se entiende que solo se está aludiendo a los hombres al hablar de empresarios y no de empresarias y al aparecer después el sustantivo gente refiriéndose a personas trabajadoras también. Por tanto, se está elevando a ciertos hombres, los empresarios, a una posición de mayor importancia social que el resto de las personas con trabajos menos valorados, donde sí se incluye a las mujeres.

\section{Debate preelectoral en el contexto formal}

En los resultados analizados en el contexto formal se aprecian algunas diferencias en cuanto al contexto informal. El candidato Garzón vuelve a presentar el lenguaje más inclusivo, más inclusivo aún que en el contexto informal, ya que aparecen más formas dobles de los sustantivos para referirse a las mujeres y referencias más neutras hacia la ciudadanía. En segundo lugar, nos encontramos con Sánchez, el cual varía notablemente su discurso debido a la referencia directa que se hace a las mujeres otorgándoles la misma relevancia que a los hombres. Además, se usan más sustantivos genéricos, por lo que el tono en este discurso se vuelve más neutro. Seguidamente, volvemos a tener dificultades para catalogar el lenguaje de Rajoy debido a que presenta un tono bastante neutro, pero usa en la mayoría de las ocasiones el masculino genérico. Pese a esto, se ha decidido catalogar su lenguaje como excluyente al entender que los sustantivos genéricos que aparecen, especialmente gente, se usan como forma de expresión muy generalizada ya que en los contextos en los que aparecen no se especifica sobre qué personas se está hablando ni tampoco se aporta alguna otra explicación que aclare a qué colectivo social se refiere. Por último, se ha decidido calificar el lenguaje de Iglesias y Rivera como lenguaje excluyente dado que se repiten las mismas características que en el contexto 
anterior; hay pocas referencias a la mujer y el masculino genérico predomina durante todo el discurso, especialmente el discurso de Rivera, en el que no aparecen citadas las mujeres en ninguna ocasión, mientras que en el de Iglesias la única ocasión en las que se les cita es para hablar de una mujer en concreto. Por lo tanto, Garzón es el representante político que muestra un lenguaje inclusivo en el contexto formal. A continuación, se muestra un resumen de los recursos léxicos más relevantes que se han nombrado con anterioridad a modo de ejemplificación:

Alberto Garzón:

11) Eso es una barrera de entrada prácticamente imposible para los hijos de las clases trabajadoras y queremos una educación que no sea para el rico sino también para los hijos de las clases trabajadoras y las hijas de las clases trabajadoras.

12) Gracias al trabajo de los voluntarios y de las voluntarias se están ganando debates.

Como podemos ver, las formas dobles aparecen de forma repetida. Sin embargo, el uso del sujeto en masculino y el masculino genérico se mantiene en el lenguaje de AG, en menor medida, pero continúa usándolo para abarcar a todas las personas, sobre todo cuando se menciona un tema económico o relacionado con el mercado laboral.

Mariano Rajoy:

13) Gracias a eso se forma la sociedad española que son más de nueve millones los que reciben una pensión.

14) A lo mejor habría que impedir el ser alcalde y diputado un poco. Podemos tener en el congreso de los diputados a gente que no tenga ninguna relación, que no pueda ser un empresario, ... [... ... que no pueda ejercer como médico.

De nuevo usa el masculino genérico y concuerda en masculino un pronombre que sustituye a un sustantivo femenino. Si seguimos analizando sus respuestas, se observa el uso considerable de gente y personas de manera muy repetitiva, que solo es sustituida por el sustantivo todos, de nuevo en masculino solamente. Esta manera de hablar se contradice un poco, ya que al ver sustantivos genéricos se espera encontrar un discurso inclusivo, que, sin embargo, se vuelve excluyente al usar solo las formas masculinas.

Pedro Sánchez:

15) En Cataluña hay una parte del malestar con la democracia que comparten con el resto de españoles y españolas.

16) Hay una prevalencia del varón sobre la mujer en un país que tiene una ley de igualdad entre hombres y mujeres. 
En esta frase, los recursos inclusivos están muy bien utilizados ya que está hablando de la sociedad visibilizando tanto a hombres como a mujeres en la misma medida. Aun así, el uso del masculino genérico sigue superando a los recursos inclusivos y a veces falta visibilidad respecto a las mujeres. El único momento en el que PS hace hincapié en diferenciar entre sexos es al hablar de la igualdad de derechos, lo que era de esperar ya que se está poniendo en relevancia las desigualdades entre mujeres y hombres.

Albert Rivera:

17) Hay muchos catalanes y muchos españoles en su conjunto a los que no les gusta la justicia politizada.

18) España es un país con el talento de una sociedad civil, con unos profesionales, que puede aspirar a ser más de lo que somos hoy.

En el primer ejemplo se podría referir al conjunto de la ciudadanía usando la palabra personas y el lugar en el que se encuentran para evitar el uso del masculino. Igualmente, en la última frase debería haber aparecido la forma femenina de unos. Resulta interesante que en esta entrevista no se menciona a las mujeres ni una sola vez. De hecho, en una pregunta en la que se le cuestiona sobre una militante de su partido que ha sido expulsada, AR no menciona ni una sola vez que es mujer, aunque la presentadora diga su nombre. AR se refiere a ella como esta persona en todo momento.

Pablo Iglesias:

19) Una persona, que es magistrado pide una excedencia, y hasta sus derechos civiles como ciudadano.

20) Los pequeños y menores autónomos y trabajadores, también los mejores magistrados, militares, personas que por su prestigio profesional pueden demostrar que pueden gobernar muy bien.

En estos ejemplos se puede observar el uso excesivo del masculino genérico en frases en las que no es estrictamente necesario. Por ejemplo, en la primera frase los adjetivos magistrado y ciudadano se han concordado en masculino a pesar de acompañar a un sustantivo femenino de persona. En el segundo ejemplo se puede observar que el sustantivo personas no actúa como un recurso inclusivo y que PI opta por usar el masculino genérico para referirse a un país en lugar de usar el nombre de este.

Por estas razones, concluimos que el representante político que usa los recursos inclusivos de las guías, el que visibiliza a las mujeres y consigue un lenguaje inclusivo en el contexto formal e informal en los discursos preelectorales es Alberto Garzón. En la siguiente tabla se resumen los resultados obtenidos del análisis preelectoral: 


\section{TABLA 1}

Resumen de los recursos más usados por cada representante político en los programas televisivos

\section{REPRESENTANTE} POLITICO

Mariano Rajoy

\section{LA CALLE PREGUNTA}

\section{LOS DESAYUNOS DE TVE}

- Escaso uso de sustantivos genéricos

- Escasa visibilidad a la mujer

- No omite el sujeto "nosotros"

- Uso frecuente del masculino genérico

- Escaso uso de sustantivos genéricos

- Escasa visibilidad a la mujer

- No omite el sujeto "nosotros"

- Uso frecuente del masculino genérico

Pedro Sánchez

- Uso habitual del masculino genérico

- Uso de formas dobles para nombrar a las mujeres en temas tradicionalmente relacionados con mujeres

- Uso de sustantivos genéricos con formas en masculino

- Uso de sustantivos genéricos - Se disminuye el uso del masculino genérico - Uso de formas dobles - Más visibilidad a la mujer - Escasa visibilidad a la mujer
- No omite el sujeto "nosotros"

- Uso de formas dobles para nombrar a las mujeres en temas tradicionalmente relacionados con mujeres

- Uso habitual del masculino genérico

- Escasa visibilidad a la mujer

- No omite el sujeto "nosotros"

- Uso de formas dobles para nombrar a las mujeres en temas tradicionalmente relacionados con mujeres - Uso habitual del masculino genérico

\begin{tabular}{lll}
\hline Pablo Iglesias & - Escasa visibilidad a la mujer & - Escasa visibilidad a la mujer \\
& - No omite el sujeto "nosotros" & - No omite el sujeto "nosotros" \\
& - Uso de formas dobles para & - Uso de formas dobles para \\
& nombrar a las mujeres en & nombrar a las mujeres en \\
& temas tradicionalmente & temas tradicionalmente \\
& relacionados con mujeres & relacionados con mujeres \\
& - Uso habitual del masculino genérico & - Uso habitual del \\
& & masculino genérico \\
\hline Alberto Garzón & - Otorga visibilidad a la mujer & - Otorga visibilidad a la mujer \\
& - Uso habitual de formas dobles & - Uso habitual de formas dobles \\
& - Omite el sujeto "nosotros" & - Omite el sujeto "nosotros" \\
& - Uso de sustantivos genéricos & - Uso de sustantivos genéricos \\
\hline
\end{tabular}

\section{Discursos postelectorales en el contexto formal}

Los resultados que muestran los análisis de los discursos postelectorales presentan variaciones significativas en comparación con los discursos preelectorales. En el caso de Rajoy aumenta el uso de los sustantivos genéricos y disminuye el masculino genérico, además de mantener el tono neutro durante todo el discurso y visibilizar, en la mayor parte del discurso, a hombres y mujeres. Respecto a Sánchez, su discurso se vuelve mucho más inclusivo al 
contar con numerosas formas dobles que citan a las mujeres y sustantivos genéricos que engloban a toda la sociedad. Además, las mujeres aparecen en asuntos de diferente índole y con la misma importancia que los hombres, por lo que ya no se aprecia el tono excluyente que encontrábamos antes de las elecciones. Por estos motivos, el discurso de Sánchez, una vez terminada la campaña, se aleja bastante del discurso que había usado durante la campaña electoral. En cuanto al discurso de Iglesias, este varía en la medida en la que se usa el masculino genérico y los sustantivos genéricos, pero mantiene el mismo lenguaje excluyente que en el discurso preelectoral. El principal motivo de este pensamiento es que los sustantivos genéricos se muestran como un recurso habitual para generalizar y citar a un gran conjunto de personas en lugar de ser un recurso inclusivo. Se sigue apreciando un tono excluyente en su discurso, además de que no se visibiliza a las mujeres ya que no se hace uso de ninguna forma doble. Igualmente ocurre en el discurso de Rivera; no se aprecian formas dobles ni menciones directas de las mujeres, sino que se sigue dando prevalencia al masculino genérico en ocasiones en las que se debería haber usado sustantivos genéricos. Asimismo, estos recursos desaparecen del discurso postelectoral y los determinantes que se emplean hacen referencia al género masculino. Finalmente, el discurso de Garzón mantiene la misma línea que en los discursos preelectorales; emplea las formas dobles de los sustantivos y los determinantes para visibilizar a la mujer, se dirige a la ciudadanía con sustantivos neutros y el número de ocasiones en las que aparece el masculino genérico es escaso en comparación con los otros representantes políticos. Por lo tanto, Garzón es el candidato político que emplea un lenguaje inclusivo también después de la campaña electoral. En las siguientes intervenciones de los representantes políticos se exponen algunos ejemplos de los recursos léxicos a partir de los cuales se han obtenido los resultados anteriormente expuestos y en la siguiente tabla se expone a modo de resumen las características del discurso de cada representante político:

Mariano Rajoy:

21) En este momento deseamos al pueblo británico un debate fructífero.

22) Creo en cualquier caso que los intereses de Europa y los intereses de España.

En estos ejemplos nos encontramos con numerosos recursos inclusivos en los que se cita a un grupo de personas nombrando a la institución para la que trabajan o el país en el que viven, por lo que se consigue aludir a unas personas en concreto de manera que se visibiliza a todas ellas. El discurso de MR en este contexto dista mucho de los dos discursos anteriores; en este se deja a un lado el masculino genérico como norma y se cambia por el neutro.

Pedro Sánchez:

23) En estos últimos meses [es importante que el gobierno] pueda apadrinar políticas tan alejadas de la sensibilidad de sus ciudadanos y ciudadanas. 
24) Su gobierno ha demostrada una completa insolidaridad con aquellos inmigrantes que más apoyo necesitan, que son precisamente los refugiados y las refugiadas.

No obstante, en este discurso escasamente se usan sustantivos genéricos como personas o gente y se mantiene el uso del masculino genérico. Aun así, el lenguaje usado durante todo el discurso es más neutro que en el apartado anterior debido a que se discute en el debate sobre el mismo tema; por lo tanto, no hay necesidad de mencionar sobre quién se está hablando, solamente cuando se cita a estas personas directamente.

Pablo Iglesias:

25) El acuerdo supone enterrar definitivamente el derecho de asilo en Europa y da la espalda a miles de personas ... [... ... esas personas no valen menos que los europeos, señorías.

26) Me hace sentir vergüenza las barbaridades que se han hecho en nuestras fronteras con seres humanos que deberían tener los mismos derechos que los españoles.

En estos ejemplos observamos que se usa el lenguaje inclusivo para citar a personas de una manera muy generalizada. En cambio, para hablar del pueblo europeo se usa el masculino genérico, en lugar de citar a Europa como conjunto de la población. El motivo de este hecho puede ser que exista un sentimiento de proximidad por parte de PI al hablar de Europa y, por tanto, no se refiera a los europeos de manera tan general ya que se incluye a sí mismo en este grupo. Por el contrario, al hablar de los refugiados puede que esa proximidad no exista y use un vocabulario más general y neutro. Esta idea queda también reflejada en el siguiente ejemplo, donde se distingue entre seres humanos y españoles. Al utilizar un sustantivo genérico y un sustantivo masculino para separar a unas personas de otras se crea un sentido de división que invisibiliza no solo a las mujeres españolas sino también al resto de personas de las que se habla específicamente. Por tanto, hubiese sido mejor cambiar las palabras escogidas para esta oración por Me hace sentir vergüenza las barbaridades que se han hecho en nuestras fronteras con seres humanos que deberían tener los mismos derechos que cualquier otro ser humano en España.

Albert Rivera:

27) Ese referéndum en Gran Bretaña es consolidar, no ya sólo una Europa a dos velocidades, que ya la tenemos, los que quieren estar en el euro y los que no están; sino consolidar una Europa a tres velocidades, los que creemos en Europa, los que creen un poquito y los que no creen nada.

28) ¿Y tenemos los europeos que asumir que somos un gigante económico y un enano político? ¿Tenemos que asumir los europeos que nos hemos hecho pequeñitos ...? ¿Esa es la Europa que queremos para nuestros hijos? 
En estos ejemplos se podría haber sustituido los que por las personas que. Asimismo, se podría cambiar la sintaxis de las preguntas retóricas y evitar usar el masculino al mismo tiempo que se evita nombrar a colectivos sociales, con lo que tendríamos un resultado neutro e inclusivo en todos los sentidos: ¿Tiene Europa que asumir que es una gran potencia económica y una potencia debilitada en política? ¿Tiene que asumir Europa que se ha empequeñecido ...? ¿Esa es la Europa que queremos para las generaciones futuras? Si comparamos este ejemplo de variación con la frase original, vemos que se mantiene el mismo significado sin mencionar a ningún colectivo y sin hacer referencia a ningún sexo, por lo que obtenemos un lenguaje inclusivo y no ofensivo.

Alberto Garzón:

29) Muchas gracias a todos y a todas. Señor Sánchez, vuelvo a la tribuna ... [... ...procede empezar con una cita dado que todo el mundo ha citado a alguien.

30) Pero también era la sonrisa de las oligarquías que están fuera de esta cámara y que, sin embargo, estaban contentas y satisfechas.

Al contrario de lo que hemos visto en algunos discursos preelectorales, AG concuerda correctamente los sustantivos femeninos genéricos con los adjetivos que siguen. De este modo, cuando se emplea una palabra femenina que alude a un grupo de personas, los adjetivos que describen a estas personas aparecen en femenino y no en masculino como en anteriores casos.

\section{TABLA 2}

Resumen de los recursos más usados por cada representante político en la sesión plenaria

\begin{tabular}{ll}
$\begin{array}{ll}\text { REPRESENTANTE } \\
\text { POLÍTICO }\end{array}$ & SESIÓN PLENARIA EN EL CONGRESO DE LOS DIPUTADOS \\
Mariano Rajoy & - Uso frecuente de sustantivos genéricos \\
& - Disminución en el uso del masculino genérico \\
& - Uso frecuente de formas dobles para incluir a la mujer \\
\hline Pedro Sánchez & - Uso frecuente de sustantivos genéricos \\
& - Disminución en el uso del masculino genérico \\
& - Uso frecuente de formas dobles para incluir a la mujer \\
\hline Albert Rivera & - Uso frecuente del masculino genérico \\
& - Escasa visibilidad a la mujer \\
& - No se usan formas dobles \\
\hline Pablo Iglesias & - Uso frecuente del masculino genérico \\
& - Escasa visibilidad a la mujer \\
& - No se usan formas dobles \\
\hline Alberto Garzón & - Uso frecuente de formas dobles para incluir a la mujer \\
& - Uso frecuente de sustantivos genéricos \\
& - Uso de “nosotros y nosotras" como sujeto \\
& - Visibilidad a la mujer \\
\hline
\end{tabular}




\section{Discusión}

Los resultados obtenidos muestran que existe un mismo patrón en el lenguaje de los cinco políticos estudiados:

1) El masculino genérico como recurso principal para abarcar a hombres y mujeres; el uso habitual de personas y gente como sustantivo genérico.

2) El empleo del sujeto nosotros, independientemente de si hablamos de una mujer o un hombre.

3) El uso de pronombres relativos neutros.

4) El hecho de citar la profesión en lugar de la institución para la que se trabaja, la cual suele ir en masculino.

5) La escasa visibilidad de las mujeres excepto cuando se trata de una temática tradicionalmente asociada con las mujeres, como la educación escolar, los cuidados a familiares o la maternidad.

De los numerosos recursos que se presentan en las guías, podemos afirmar que la mayor parte de estos aparecen en algunas ocasiones en el lenguaje de los representantes políticos analizados, aunque no en la misma medida y dependiendo del representante político. Respecto a los recursos gramaticales, nos encontramos tanto con la omisión del sujeto y con cambios en la sintaxis, mientras que en los recursos léxicos observamos numerosos sustantivos genéricos, formas dobles y abreviadas y determinantes y artículos omitidos. Asimismo, no encontramos ejemplos claros en los que se cosifique a la mujer; sin embargo, no se le concede la misma importancia al trabajo que realizan; solamente, como hemos mencionado anteriormente, se le concede en trabajos asociados con la mujer. No obstante, estos recursos inclusivos tienen una presencia reducida en el análisis del lenguaje y, además, no están presentes en todos los candidatos.

Como manera de resumen, hemos elaborado una tabla con los resultados de cada contexto antes y después de las elecciones según cada candidato (véase la tabla en la página siguiente).

Además de los pequeños análisis que se han descrito con cada ejemplo, hay más variables que se deben analizar, como, por ejemplo, la ideología política, la intención comunicativa y el tipo de discurso. Como se ha podido apreciar en los resultados, los representantes políticos que más han usado formas no inclusivas del lenguaje han sido aquellos que pertenecen a partidos de derechas, Partido Popular y Ciudadanos, mientras que el candidato que pertenece al partido más de izquierdas, Izquierda Unida, tiene el discurso más inclusivo de todos. Sin embargo, no podemos afirmar que la ideología sea un factor importante, ya que Pablo Iglesias, el candidato de Podemos, un partido político de izquierdas, muestra un discurso muy alejado de lo que aquí se considera inclusivo, y, además, Pedro Sánchez, del Partido Socialista, varía su 


\section{TABLA 3}

Resumen de los resultados de cada representante político en los contextos analizados

\begin{tabular}{llll}
$\begin{array}{l}\text { REPRESENTANTE } \\
\text { POLítico }\end{array}$ & $\begin{array}{l}\text { CONTEXTO INFORMAL } \\
\text { PREELCTORAL }\end{array}$ & $\begin{array}{l}\text { CONTEXTO FORMAL } \\
\text { PREELCTORAL }\end{array}$ & $\begin{array}{l}\text { CONTEXTO FORMAL } \\
\text { POSTELECTORAL }\end{array}$ \\
Mariano Rajoy & No usa lenguaje inclusivo & No usa lenguaje inclusivo & Usa lenguaje inclusivo \\
\hline Pedro Sánchez & No usa lenguaje inclusivo & Usa lenguaje inclusivo & Usa lenguaje inclusivo \\
\hline Albert Rivera & No usa lenguaje inclusivo & No usa lenguaje inclusivo & $\begin{array}{l}\text { No usa lenguaje } \\
\text { inclusivo }\end{array}$ \\
\hline Pablo Iglesias & No usa lenguaje inclusivo & No usa lenguaje inclusivo & $\begin{array}{l}\text { No usa lenguaje } \\
\text { inclusivo }\end{array}$ \\
\hline Alberto Garzón & Usa lenguaje inclusivo & Usa lenguaje inclusivo & Usa lenguaje inclusivo \\
\hline
\end{tabular}

discurso dependiendo del contexto. Si tenemos en cuenta la intención comunicativa de los representantes políticos, entendemos que su intención es de persuadir al oyente y convencerlo de que le otorgue su voto; por lo tanto, el uso de recursos inclusivos en sus discursos se puede deber a que quieren obtener el voto femenino, ya que este representa una gran parte de España, más que visibilizar a las mujeres en el lenguaje. En cuanto al tipo de discurso, no se aprecia que el lenguaje formal e informal sean también factores significantes para el lenguaje inclusivo. Si observamos los resultados en la tabla anterior, solo dos de los cinco candidatos varían su discurso al pasar del contexto informal al contexto formal. La razón de este cambio se podría deber a que los discursos en el contexto formal se habían elaborado previamente y se estaba siguiendo un guion para responder a las preguntas formuladas mientras que en el contexto informal los políticos no habrían tenido la oportunidad de preparar sus respuestas, por lo que el lenguaje estaba menos elaborado y pensado.

\section{Conclusiones}

Tras la presentación de los resultados y la discusión sobre estos, queremos recordar las hipótesis con las que comenzábamos este trabajo con el fin de comprobar si eran ciertas y si hemos sido capaces de responder a las preguntas que nos hacíamos antes de obtener los resultados.

Con la primera pregunta de investigación que formulábamos se quería averiguar si los representantes políticos utilizaban siempre el mismo tipo de lenguaje antes y después de las elecciones. Como hemos podido comprobar, la respuesta a esta pregunta es negativa, hecho que se puede confirmar si examinamos el número de variaciones que se han presentado en el lenguaje usado durante las entrevistas y los debates en el Congreso. Seguidamente, en la segunda pregunta de investigación se pretendía responder a si influiría el contexto en el lenguaje de los representantes, es decir, si el lenguaje inclusivo sería más frecuente antes de 
las elecciones para poder así captar más votantes y obtener un mejor resultado. Los resultados obtenidos de los análisis nos muestran que el contexto no influye en el lenguaje, y, por tanto, que el hecho de estar en campaña electoral no interfiere en la forma en la que se usa el lenguaje. A continuación, en la tercera pregunta de investigación se quería averiguar si el tema tratado es un factor relevante para optar por un lenguaje inclusivo. Como se ha visto en los resultados, la respuesta es positiva; los representantes políticos tienden a nombrar directamente a las mujeres cuando se tratan temas como el cuidado de los hijos, la educación escolar o el cuidado de personas con necesidades especiales.

Respecto a las hipótesis, la primera de ellas sugería que todos los representantes políticos usarían un lenguaje inclusivo mayormente antes de las elecciones, lo cual ha resultado ser solo cierto para el candidato del PSOE, Pedro Sánchez, ya que él sí varía su lenguaje al encontrarse en una situación formal, haciéndolo más inclusivo. No obstante, el resto de los candidatos no comparten esta característica, por lo que consideramos que esta hipótesis no es cierta. La siguiente hipótesis que planteábamos proponía que el lenguaje usado por los representantes políticos variaría después de las elecciones, pero, al igual que en la hipótesis anterior, esta hipótesis tampoco es cierta, ya que el único candidato que varía su lenguaje después de las elecciones es Mariano Rajoy. Para finalizar, la última hipótesis sugería que el lenguaje inclusivo sería propio del contexto formal y no tendría lugar en el contexto informal. Sin embargo, de igual modo que en las hipótesis anteriores, los resultados muestran que esta hipótesis es errónea, puesto que el único candidato que varía su lenguaje según el contexto es Pedro Sánchez.

Después de haber valorado los resultados, es importante decir que con estos resultados no se quiere transmitir la idea de que los representantes políticos que se han analizado están en contra del feminismo o del lenguaje inclusivo. De hecho, como se puede observar en sus discursos, todos y cada uno de ellos afirman estar a favor de la igualdad y de buscar formas de acabar con el sexismo que existe en la sociedad. Por eso queremos recalcar que este estudio solamente analiza el uso de las palabras con las que se expresan en determinados contextos y no su forma de pensar. Esta idea también es argumentada por Olalla Martínez (2015) en el discurso de los y las militantes de Podemos: "en el discurso de Podemos no se observa un tratamiento sistemático y homogéneo del lenguaje inclusivo. Pese a que de forma explícita sus miembros ponen de manifiesto la cuestión de género y su preocupación por la igualdad y por la visibilidad de la mujer" (2015: 31). Es muy probable que la razón por la cual los candidatos no usan el lenguaje inclusivo es que es una corriente contemporánea que aún no se ha estabilizado en la sociedad, además de que hay personas e instituciones, como la RAE, que se oponen al lenguaje inclusivo, por lo que usan el masculino genérico como norma para expresarse en castellano sin ningún tipo de connotación machista. A pesar de esto, hemos comprobado, gracias a las guías y al discurso de algunos candidatos, que es posible expresarse usando el lenguaje inclusivo y que es una gran herramienta para conseguir una sociedad más igualitaria. 


\section{Bibliografía citada}

Ajuntament de Terrassa, 2004: Manual de comunicación no sexista, 21 de junio de 2004.

Alcaidea L., Esperanza R., 2009: “El ‘yo' de los políticos: ¿cuestión de género?”, Discurso y Sociedad 6 (1), 5-20 [disponible en http//www.dissoc.org, fecha de consulta: 22 de junio de 2018].

Asociación Cultural Simone de Beauvoir, 2006: Guía para la utilización no sexista del lenguaje, Madrid: Ayuntamiento de Torrejón de Ardoz. Concejalía de la Mujer.

BengoecheA, Mercedes, 2002: Guía para la revisión del lenguaje desde la perspectiva de género, Madrid: Universidad de Alcalá.

Bosque, Ignacio, 2012: "Sexismo lingüístico y visibilidad de la mujer", Boletín de Información Lingüística de la Real Academia Española 1, 1-18.

Enciclopedia Online, 2018: "Lenguaje formal e informal” [disponible en https://enciclopediaonline.com/es/lenguaje-formal-e-informal/, fecha de consulta: 27 de febrero de 2019].

Francesconı, Armando, 2012: "El sexismo en el lenguaje político español e italiano", Nómadas. Revista Crítica de Ciencias Sociales y Jurídicas 34.

Fuentes R., Catalina, 2013: "Argumentación, (des)cortesía y género en el discurso parlamentario", Tonos Digital: Revista Electrónica de Estudios Filológicos 25.

LLEDó, Eulàlia, 2004: "Nombrar a las mujeres, describir la realidad: la plenitud del discurso: claves para tratarse bien en la lengua, claves también para caer en la cuenta cuando esto no ocurre y entender por qué y cómo" en Uso del Lenguaje en el Mundo Laboral, País Vasco: Emakunde. Eusko Jaurlaritza / Gobierno Vasco, Fondo Social Europeo, 1-37.

Lledó, Eulàlia, 2012: Lenguaje inclusivo, Barcelona: Fundació Factor Humà. Unidad de Conocimiento - Lenguaje Inclusivo.

Martinez, Olalla, 2015: Sexismo lingüístico y discurso político: análisis del lenguaje inclusivo en el discurso de Podemos. Tesis de Grado en Tradución e Interpretación, Facultade de Filoloxía e Tradución, Universidade de Vigo.

Moreno, Damián. 2012: "Los alcaldes y alcaldesas: referencia a personas y género en el lenguaje parlamentario andaluz", Discurso y Sociedad 6 (1), 216-233 [disponible en http//www.dissoc. org, fecha de consulta: 22 de junio de 2018]. 
nOMBra: Comisión Asesora sobre Lenguaje del Instituto de la Mujer (Carmen Alario y otros), 1995: Nombra. En femenino y en masculino, Madrid: Instituto de la Mujer.

Pérez-Reverte, Arturo, 2016: "No siempre brilla y da esplendor" en Patente de Corso [disponible en https://www.xlsemanal.com/firmas/20161003/reverte-opinion-rae.html, fecha de consulta: 22 de junio del 2018].

Real Academia de la Lengua Española: "Los ciudadanos y las ciudadanas, los niños y las niñas" en "Preguntas frecuentes" [disponible en www.rae.es, fecha de consulta: 22 de junio de 2018].

RuIz, Jesús, 2016: "Los académicos y las académicas discuten sobre sexismo lingüístico", El País [disponible en http://elpais-com/cultura/2016/10/11/actualidad/1476204624_012306.html, fecha de consulta: 22 de junio del 2018].

UNED, 2012: Documental Historia Crítica del Feminismo Español.

UNESCO, 1990: Recomendaciones para un uso no sexista del lenguaje.

Universitat Autònoma de Barcelona, 2011: Guía para el uso no sexista del lenguaje (escrita originalmente en catalán por Heura Marçal, Fiona Kelso y Mercè Nogués; traducción al castellano de Gemma Lozano), Servicio de Lenguas y Observatorio para la Igualdad, Barcelona: Universitat Autònoma de Barcelona. 